Published in final edited form as:

Health Place. 2020 November ; 66: 102442. doi:10.1016/j.healthplace.2020.102442.

\title{
The Role of Neighborhood Stressors on Cognitive Function: A coordinated Analysis
}

\author{
Elizabeth Muñoz, Ph.D. ${ }^{1,2}$, Stacey B. Scott, Ph.D. ${ }^{3}$, Robin Corley, Ph.D. ${ }^{4}$, Sally J. \\ Wadsworth, Ph.D. ${ }^{4}$, Martin J. Sliwinski, Ph.D. ${ }^{5}$, Chandra A. Reynolds, Ph.D. ${ }^{1}$ \\ ${ }^{1}$ Department of Psychology, University of California, Riverside, Riverside, CA \\ ${ }^{2}$ Department of Human Development and Family Sciences, University of Texas at Austin, Austin, \\ TX \\ ${ }^{3}$ Department of Psychology, Stony Brook University, Stony Brook, NY \\ ${ }^{4}$ Institute for Behavioral Genetics, University of Colorado at Boulder, Boulder, CO \\ ${ }^{5}$ Department of Human Development and Family Studies and Center for Healthy Aging, \\ Pennsylvania State University, University Park, PA
}

\begin{abstract}
The objective of this study was to evaluate the association between perceived neighborhood stressors, encompassing negative perceived neighborhood characteristics, and specific cognitive abilities in adulthood. We conducted a coordinated analysis across three studies of adults in the United States and found that perceived neighborhood stressors were consistently associated with poorer performance on attention-demanding cognitive tasks. We specifically found that perceived neighborhood stressors were associated with lower performance in spatial abilities, working memory, and executive function but not perceptual speed, and that the effect was most consistent for lower perceived neighborhood safety followed by lower perceived aesthetic quality, greater perceived neighborhood crime, and lower perceived neighborhood cohesion. These results highlight the importance of the psychosocial neighborhood context for cognitive health in adulthood.
\end{abstract}

\section{Keywords}

Perceived Neighborhoods; Neighborhood Stressors; Cognitive Health; Adulthood

Cognitive health is imperative for retaining functional independence and maintaining engagement in work and social environments throughout adulthood (Krueger et al., 2009).

Address correspondence regarding this manuscript to: Elizabeth Muñoz, 108 E Dean Keeton St., Austin, TX 78712, Elizabeth.Munoz@austin.utexas.edu.

Publisher's Disclaimer: This is a PDF file of an unedited manuscript that has been accepted for publication. As a service to our customers we are providing this early version of the manuscript. The manuscript will undergo copyediting, typesetting, and review of the resulting proof before it is published in its final form. Please note that during the production process errors may be discovered which could affect the content, and all legal disclaimers that apply to the journal pertain.

Conflict of Interest: none declared 
Understanding how environments in which individuals develop and live may influence cognitive health outcomes is of interest to the field of cognitive development and aging. Specifically, research has been focused on understanding how neighborhoods, defined in various ways, relate to cognitive health outcomes. Prior research has shown that the social (e.g., crime/violence; social cohesion), physical (e.g., aesthetics; decaying infrastructure), and economic (e.g., neighborhood socioeconomic status) characteristics of neighborhoods are associated with physical and mental health outcomes such as heart disease (Augustin et al., 2008), mortality (Bosma, 2001; Sampson et al., 2002), disability (Pruchno et al., 2012), and depressive symptoms (Aneshensel et al., 2007). Comparatively fewer studies have examined associations between neighborhood profiles and cognition in adulthood, but those that have generally find that poor social, economic, and physical neighborhood features are associated with reduced cognitive performance. For example, those who reside within neighborhoods characterized by lower education and SES, and greater deprivation of resources (e.g., barriers to housing services) tend to perform more poorly on several standardized cognitive tasks compared to their counterparts (Aneshensel et al., 2011; Lang et al., 2008; Wight et al., 2006; Zeki Al Hazzouri et al., 2011). These findings remain after accounting for individual-level risk factors, such as household income or educational attainment (Clarke et al., 2015). Living in low quality neighborhoods has also been shown to increase vulnerability to the effects of hazardous materials (e.g., lead exposure (Glass et al., 2009). Similarly, adverse neighborhood characteristics appear to compound vulnerability among individuals at genetic risk for cognitive impairment (e.g., presence of APOE-e4 genotype Boardman et al., 2012).

With the exception of a few studies (Estrella et al., 2020; Lee \& Waite, 2018; Zaheed et al., 2019), most evidence for the association between neighborhoods and cognition has come from studies assessing the effects of objective and structural components of neighborhoods such as neighborhood SES and unemployment rates obtained from publicly available data (Wu et al., 2015). Although subjective perceptions may result from the objective and structural characteristics of an individual's neighborhood and have more proximal implications for the way in which neighborhoods relate to health outcomes (Cohen et al., 1983; Lazarus \& Folkman, 1984). There has been less evidence documenting their associations with cognition. A recent study by Lee and Waite (2018) evaluated both objective and subjective measures of neighborhoods in relation to global cognition and found that the subjective neighborhood measures more consistently predicted lower cognitive performance among an adult sample from 57 to 85 years of age. Using data from the Health and Retirement Study (ages 51 - 101), Zaheed and colleagues (2019) showed that greater perceptions of physical disorder were associated with lower performance in a measure of episodic memory, and greater perceived neighborhood social cohesion was associated with better performance in a measure of verbal fluency. Estrella and colleagues (2020) recently reported that greater perceived neighborhood problems were associated with lower global cognition and memory in Hispanic/Latina women. They also found an unexpected negative association between greater perceived social cohesion and tasks of executive abilities. In line with these recent results, we aimed to evaluate whether specific self-reported neighborhood domains or features (e.g., perceived social cohesion, perceived neighborhood problems, etc.) were associated with specific cognitive function abilities. 
Individual perceived neighborhood domains may give rise to, or reduce, psychological distress through multiple mechanisms. For example, greater perceptions of neighborhood cohesion promote feelings of trust and unity with neighbors (Sampson, 2003) and confer the benefits of social support on cognitive health outcomes by promoting social and physical activities (Fisher et al., 2004) and buffering the adverse effects of stress (Cohen \& Wills, 1985). On the contrary, greater perceived neighborhood crime and lower feeling of safety within one's neighborhood may offset the benefits of neighborhood cohesion (Choi \& MatzCosta, 2018). Perceptions of aesthetic quality incorporate views of how pleasant and attractive one's neighborhood is and lower perceptions in this domain have been associated with lower physical activity (Su et al., 2014) and poor health outcomes (Malambo et al., 2017). Poorer neighborhood perceptions are also associated with appraised severity of, and emotional responses to, stressors in daily life. Scott and colleagues showed that greater perceived neighborhood violence was associated with greater perceived severity of everyday stressors and that greater perceived violence, lower perceived safety, and lower perceived aesthetic quality were associated with greater negative affective responses to these stressors (Scott et al., 2018). This body of evidence indicates that poorer neighborhood perceptions may shape cognitive health through behavioral and emotional pathways and that these perceptions exacerbate psychological responses when stressors occur.

Poorer perceptions of neighborhood characteristics are theorized to give rise to a heightened sense of vigilance, alarm, or threat that activates the stress response and leads to adverse health outcomes over time if such responses are chronic or consistent (Augustin et al., 2008; Glass et al., 2009). The associated heightened sense of threat or worry resulting from adverse perceived neighborhood conditions, may also disrupt attentional processing needed for completion of the cognitive tasks at hand in the short-term. In the long-term, these psychosocial neighborhood characteristics, henceforth 'perceived neighborhood stressors', may therefore promote individuals' chronic stress and lead to adverse cognitive health outcomes (Augustin et al., 2008; Diez Roux \& Mair, 2010; Lupien \& Lepage, 2001;). In the current study, we focused on the associations between specific domains of perceived neighborhood stressors (i.e., perceived neighborhood violence, social cohesion, safety, and aesthetic quality) and cognitive performance. We specifically focused on fluid cognitive abilities related to executive and attention-demanding cognitive tasks such as working memory and processing speed, compared to tasks related to crystalized abilities, that are more reliant on experience or do not begin to show declines until older age, such as verbal or episodic memory abilities (Horn \& Cattell, 1967; Nyberg et al., 2003). Based on work in younger individuals (McCoy et al., 2015) and the research reviewed above, we speculated that greater perceived neighborhood stressors occupy attentional processing resulting in disrupted performance in attention demanding cognitive tasks.

Most prior evaluations of perceived neighborhood stressors and cognition have focused on older individuals (Lee \& Waite, 2018; Zaheed et al., 2019), but because cognitive decline may begin before old age, identifying early risk factors requires evaluating associations before or during midlife (Hughes et al., 2018). Further motivating an examination before old age is evidence of the negative associations between perceived neighborhood stressors and cognitive function earlier the lifespan, in adolescence, (e.g., Boyle et al., 2007) indicating that perceived neighborhood-related shifts in cognitive status may already be occurring 
before old age. We therefore sought to evaluate associations between neighborhood stress and cognitive function in three studies of adults with age ranges starting at the cusp of midlife. A coordination of analyses across multiple studies also maximizes comparability of results, as well as replication and validity of results (Hofer \& Piccinin, 2009). Thus, our goal for this study was to evaluate associations between perceived neighborhood stressors and tasks of fluid cognitive abilities across three studies of adults. Because there may be variation in neighborhood contexts according to a study's specific recruitment strategy and location, we chose to test our hypotheses in three studies across different geographical contexts with comparable measures of perceived neighborhood stressors. Data for one of the studies were collected in a prescribed area in New York, USA (ESCAPE; Scott et al., 2015) another that began in Colorado, USA but with participants currently living across the country (CATSLife; Wadsworth et al., 2019), and one that was designed as a national survey of midlife in the United States (MIDUS; Ryff et al., 2007). The studies incorporated psychosocial neighborhood measures that captured multiple domains of perceived neighborhood stressors, such as feelings of safety in one's neighborhood, aesthetic quality, and social cohesion, and they also included measures of fluid or attention-demanding cognitive abilities (e.g., working memory). Based on prior evidence, we predicted that greater levels of perceived neighborhood stressors, characterized by lower self-reported neighborhood perceptions, would be associated with poorer performance on fluid, attentiondemanding tasks and that this finding would be replicated across the three studies of interest.

\section{Method}

\section{CATSLife Sample}

The Colorado Adoption/Twin Study of Lifespan behavioral development and cognitive aging (CATSLife) is a longitudinal study of lifespan behavioral development and cognitive aging that merges two developmental studies with decades of previously collected data, the Colorado Adoption Project and the Longitudinal Twin Study with a new assessment of participants as they approach midlife. Details on this study can be found in Wadsworth and colleagues (2019) and the study's protocol was approved by its respective institutional review board. Briefly, CATSLife's study procedures consist of an online survey that encompasses measures of demographics, relationships, health, well-being, and environments, including the perceived neighborhoods questionnaire, and an in-person assessment during which biomarker, health, and cognitive function data are collected.

Data collection for this study is ongoing and the current analysis focused on data from 1,278 individuals collected before April 2020. Inclusion criteria for the current analyses were: (1) available data on at least one cognitive outcome of interest; (2) available data on perceived neighborhood stressors. Descriptive characteristics of the 1,216 individuals included in this study are presented in Table 1.

Perceived Neighborhood Stressors.-In CATSLife, twenty-one questions were included that asked participants to indicate from a list of problems, whether each was a problem in their neighborhood on a three-point scale from Not a problem to a Big problem. Sample questions included: "high unemployment," "abandoned houses," and "police not 
available when needed." One question from the PhenX Activity toolkit was also included as it tapped neighborhood safety (Sallis et al., 2009; Sallis \& DeBourdeaudhuij, 2004). This question asked participants to indicate on a four-point scale the extent to which they strongly agreed (4) to strongly disagreed (1) to the following item: "The crime rate in my neighborhood makes it unsafe to go on walks at night". All questions were scored accordingly so that high scores indicated higher neighborhood problems or stressors (for a complete list of items see Supplementary Table 2).

Cognitive Assessments.-During the in-person assessment, participants completed measures of perceptual speed and spatial abilities. The specific cognitive abilities battery incorporating these measures was based on the Hawaii Family Study of Cognition (DeFries et al., 1978, 1981; Wadsworth et al., 2019). The z score standardized cognitive task scores were added together to form a cognitive ability composite and this composite was then $\mathrm{z}$ score standardized resulting in a mean of zero and standard deviation of one for both the cognitive task domains.

Perceptual speed.: Assessed via the Colorado Perceptual Speed Test and a Subtraction and Multiplication task. The dependent measure was the number of correct responses per trial.

Colorado Perceptual Speed Task.: Participants were asked to identify a target string of unpronounceable letter and number strings among three foils. This was a two-part test with 60 seconds dedicated for each part containing a maximum of 30 trials per block (60 trials total).

Subtraction and Multiplication.: Participants were asked to complete a series of subtraction and multiplication tasks as rapidly and accurately as possible. This was a two-part test with 120 seconds dedicated for each part with a maximum of 60 trials per block (120 trials total).

Spatial ability.: Assessed via a Paper Foam Board task and a Cards rotation task.

Paper Form Board task.: One geometric figure was presented on the left and two to four pieces on the right that would result if the intact figure were cut into parts. The participant was instructed to draw lines on the complete figure on the left, indicating where it should be cut to form the parts to the right. There were 28 trials and the dependent measure was the number of entirely correct responses for each trial.

Card Rotations task (ETS).: An irregularly shaped stimulus figure was presented to the left and eight variations of the same figure to the right on the same row. Some of the figures on the right were the same as on the left, but were rotated on the plane of the page and others were flipped over so that they were mirror images of the stimulus figure. Participants were asked to determine if each of the eight figures on the right were rotated or a mirror image of the stimulus figure. This was a two-part test with 14 trials per block. The dependent measure was the total number of correct responses.

Covariates.-Demographic covariates included: age at time of assessment, sex, race and ethnicity, and highest degree of education $(0=$ less than High School to $4=$ more than 
college). Socioeconomic variables included: employment status (employed vs. not employed), marital status $(0=$ married, $1=$ widowed, $2=$ divorced, $3=$ separated, $4=$ never married/other), and financial strain as a proxy for income as not all three studies had income data available. Lastly, we include measures of self-reported mental and physical health.

Financial strain.: Participants responded on a scale from 1 (Feel comfortable/Never worry) to 10 (Feel overwhelmed/Worry all the time) to the following item: "How often do you worry about being able to meet monthly living expenses" from the InCharge Financial Distress/Financial Well-being Scale (Prawitz et al., 2006). Higher scores indicated greater financial strain.

Depressive symptoms.: Measured via four items from general depression symptoms scale from the Mood and Anxiety Symptoms Questionnaire (Wardenaar et al., 2010). Participants responded from Very slightly (1), to Extremely (5) the extent to which they experienced a list of items in the past week (e.g., felt like a failure; was disappointed in myself). A sum score of total depressive symptoms was used were higher scores indicated greater depressive symptoms.

Self-reported physical health.: Participants responded to one item: "In general would you say your health is..." via the following (1) Excellent, (2) Good, (3) Fair, (4) Not well, (5) Poor (Rhea et al., 2013). Responses were recoded accordingly so that higher scores indicated better self-reported health.

\section{ESCAPE Sample}

The Effects of Stress on Cognitive Aging, Physiology and Emotion study (ESCAPE) is a measurement burst study of daily experiences of stress and daily cognition. This study was approved by its respective institutional review board and details can be found in Scott et al. (2015). Briefly, 256 participants were recruited through letters and phone calls using systematic probability sampling of New York City Registered Voter Lists for one specific zip code in an area of Bronx, New York (10475). Eligibility criteria included: participants must be between 25 and 65 years of age, ambulatory, fluent in English, without visual impairment, and being a resident of Bronx County The study's protocol consisted of baseline paper surveys that contained demographic questions, personality, stress, physical and mental health, and a perceived neighborhoods questionnaire, and an in-lab visit where cognitive function was assessed. Following the in-lab assessment, participants also completed 14 days of ecological momentary assessments that were not used in the current analysis. Baseline survey and in-lab cognitive assessment data from the first assessment wave were used in this study. Inclusion criteria for the current analyses were: (1) available data on at least one cognitive outcome of interest; (2) available data on perceived neighborhood stressors. Descriptive characteristics of the 253 individuals included in this study are presented in Table 1.

Perceived Neighborhood Stressors.-The perceived neighborhood measure in ESCAPE was adapted from Mujahid and colleague's larger multi-domain measure (Mujahid et al., 2007). The ESCAPE study included measures that tapped into the following domains: 
aesthetic quality, social cohesion, safety, violence, and availability of healthy foods. For the violence subscale, participants rated the frequency in which four events related to neighborhood violence (i.e., mugging, fight with a weapon, gang fight, sexual assault) occurred during the last six months from often (1) to very rarely (4); items were reverse scored such that higher scores represented more frequent perceived violence. For the aesthetic quality, social cohesion, and safety domains, participants rated the extent to which they agreed with statements on a strongly agree (1) to strongly disagree (5) scale for such items as "There is a lot of trash and litter in my neighborhood," "People in my neighborhood can be trusted," and "I feel safe walking in my neighborhood at night" from the three domains, respectively. All questions were scored accordingly so that high scores indicated higher perceived neighborhood stressors or problems in the specific domains (e.g., lower perceived safety). See Supplementary Table 3 for a complete list of items across all perceived neighborhood stressor domains in ESCAPE.

Cognitive Assessments.-Participants were individually tested by a trained researcher in a quiet testing room in a single session. Consistent with the procedure used for CATSLife, a standardized sum score with a mean of zero and a standard deviation of one was computed for each cognitive domain listed below.

Perceptual speed.: Assessed via a symbol match, a letter match (Wechsler, 1996), and a number match (Salthouse, 1996) through which participants were required to make speeded perceptual comparisons across items presented in a visual display. The median reaction time in seconds for correct trials was used as the dependent measure whereby higher scores indicate slower reaction times and thus poorer functioning in this cognitive domain.

Symbol match.: Participants were presented with six symbol pairs at the top of the screen and were presented with a comparison symbol pair at the bottom of the screen. Participants were asked to decide as quickly as possible whether the target pair at the bottom of the screen exactly matched one of the pairs at the top of the screen. Participants completed 70 trials of this task.

Letter match.: Participants were presented with two-letter strings and they were asked to determine whether the strings were the same or different as quickly as possible. Strings were three, six, or nine, characters long and mismatched strings varied by one character. Participants completed 70 trials with one break at trial 35 .

Number match.: Participants were presented with two number strings and were asked to indicate whether the strings were the same or different as quickly as possible. Strings were three, six, or nine items long and participants completed 70 trials

Working memory.: Assessed via an operation span, counting span (Conway et al., 2002), and a backward letter span task (Waters \& Caplan, 2003).

Counting span.: Administration of this task followed the procedures described in Engle et al. (1999). Participants were asked to memorize the number of targets in a series of displays that included dark blue circles (targets) and dark blue squares (color distractors) and light 
blue circles (shape distractors). Each display was initiated by a tester. Participants counted the number of dark blue circles aloud and repeated the digit corresponding to the final count. The number of targets per display varied from two to six, with three trials of each. After two to six displays, a recall cue was presented, at which point participants reported the number of targets in each of the previous displays, in the serial order in which they occurred. The dependent measure was the total number of target counts recalled in the correct order.

Operation span.: Participants were asked to verify equations aloud while trying to remember letters. As with the counting span, after a series of numeric equations and letters were presented, participants were prompted to recall all the letters from that series. The number of equation and letter pairs per series varied from two to five with three series of each length presented in a fixed random order. The dependent measure was the total number of letters recalled in the correct order.

Backward span.: Participants saw a series of letters presented one at a time for one second each. At the end of each series, participants recalled all the letters they saw in reverse order. The number of letters in each series varied from three to eight and participants attempted two trials each length for a total of 12 trails. The dependent measure was the total number of items for trials that were recalled in the correct order.

Covariates.-Demographic covariates included: age at time of assessment, sex, race and ethnicity, and highest level of education completed $(0=$ less than High School to $4=$ more than college). Socioeconomic variables included: employment status (employed vs. not employed $)$, marital status $(0=$ married, $1=$ widowed, $2=$ divorced, $3=$ separated, $4=$ never married/other), and financial strain. Lastly, we include measures of self-reported mental and physical health.

Financial strain.: Participants responded on a scale from 1 (Not at all true) to 3 (Very true) to: "You find it difficult to pay monthly bills" (Turner \& Wheaton, 1997) whereby higher scores indicated greater financial strain.

Depressive symptoms.: Measured via eight items from the PROMIS emotional distress short form. Participants responded from Never (1) to Always (5) the extent to which items (e.g, helpless, unhappy) best described how they felt in the past seven days with higher scores indicating more depressive symptoms (PROMIS Health Organization, 2012a).

Self-reported physical health.: Participants responded to one item: "In general, would you say your health is..." via the following (1) Poor, (2) Fair, (3) Good, (4) Very good, (5) Excellent, where high values indicated better perceived health (PROMIS Health Organization, 2012b).

\section{MIDUS 2}

The second wave of the Midlife in the United States (MIDUS 2) was collected between 2004 and 2006 (http://www.midus.wisc.edu/midus2/). Details about MIDUS 2 can be found in Ryff et al. (2007). Broadly, the focus of this study was to evaluate the role of behavioral and psychosocial factors in physical and mental health. As a follow-up to the first wave of data 
collection for MIDUS, MIDUS 2 assessments mirrored those of the baseline wave with the inclusion of new assessments of cognitive function via telephone. Participants were recruited to the first wave of MIDUS via random digit dialing of United States households having at least one telephone in the contiguous 48 states between 1995-1996; the follow-up assessment in the current analyses began 9 years later and involved $75 \%(\mathrm{~N}=3,615)$ of the original (MIDUS) sample (Hughes et al., 2018). Perceived neighborhood data were collected via self-administered mailed-in questionnaires that also included other psychosocial and behavioral assessments. Cognitive function was assessed via telephone using the Brief Test of Adult Cognition by Telephone (Tun \& Lachman, 2006). Inclusion criteria for the current analyses were: (1) available valid data on at least one cognitive outcome of interest; (2) available data on perceived neighborhood stressors. Descriptive characteristics of the 3,198 individuals meeting inclusion are presented in Table 1.

Perceived Neighborhood Stressors.-MIDUS 2 included twelve questions that asked participants to indicate the extent to which a series of statements described their situation related to the neighborhoods they lived in. Participants answered on a 4-point scale from (1) A lot to (4) Not at all to items such as "I feel safe being out alone in my neighborhood during the daytime" or "I could call on a neighbor for help if I needed it." All questions were scored so that higher sores indicated more perceived neighborhood-related stressors or problems (see Supplementary Table 4 for complete list of items).

Cognitive Assessments.-We used the recommended z score standardized (Mean $=0$, $S D=1$ ) factor score of executive functioning using tasks from the Brief Test of Adult Cognition by Telephone (for details see Lachman \& Tun, 2008; Tun \& Lachman, 2006).

Executive functioning.: The standardized executive functioning composite score made publicly available is a z score standardized score of five subtests (Agrigoroaei \& Lachman, 2011): backward digit span (the highest digit span achieved when repeating strings of digits in reverse order), verbal fluency (the number of words produced in 60 seconds from the animal category), inductive reasoning (completing a pattern in a series of five numbers), processing speed (the number of digits produced in 30 seconds by counting backward from 100), and attention switching and inhibitory control (using the Stop and Go Switch Task).

Covariates.-Demographic covariates included: age at time of the phone interview, sex, race and ethnicity, and highest year of education $(0=$ less than High School to $4=$ more than college). Socioeconomic variables included: employment status (employed vs. not employed), marital status ( $0=$ married, $1=$ widowed, $2=$ divorced, $3=$ separated, $4=$ never married/other), and financial strain. Lastly, we include measures of self-reported mental and physical health.

Financial strain.: Participants responded on a scale from 1 (Very difficult) to 4 (Not at all difficult) to the following item: "How difficult is it for you to pay your monthly bills?" and was recoded so that high scores represented greater financial strain.

Depressive symptoms.: Measured via seven items that asked respondents to determine whether or not (Yes/No) they had experienced a list of behaviors for two weeks in the past 
year when they felt sad, blue or depressed (e.g., felt down on yourself, no good, or worthless). A total score of the "Yes" responses was computed whereby higher scored indicated greater depressive symptoms (Wang et al., 2000).

Self-reported physical health.: Participants responded to one item: "Would you say your physical health is..." via the following (1) Excellent, (2) Very good, (3) Good, (4) Fair, (5) Poor, and was recoded so that high values indicated better perceived health.

\section{Analytic Approach}

To replicate the neighborhood domains from the perceived neighborhood measure used in ESCAPE (i.e., safety, aesthetic quality, cohesion, healthy foods, violence), we conducted exploratory factor analyses in the CATSLife and MIDUS 2 neighborhood measures using Mplus version 7.4, accounting for non-independence using the CLUSTER statement (Muthén \& Muthén, 1998 - 2012) with oblique rotation and the maximum likelihood estimator. Supplementary Table 1 shows the model fit indices for the four- versus a fivefactor solutions and Supplementary Tables 2 and 4 present the loadings of the items on the factors along with the factor correlations. The items in the CATSLife measure loaded on factors representative of safety, crime, aesthetic quality, disorder, and lack of services. Although fit indices indicated a better fit for a five-factor solution in MIDUS, the factor structure for a four-factor solution was theoretically sound as the four items loading on the pride constructs, that was ultimately not used, split into two factors in the five-factor model. The items in MIDUS loaded on factors representative of safety, aesthetic quality, cohesion, and pride. The factor loading for the ESCAPE sample are also presented in Supplementary Table 3 for ease of comparability. Table 1 presents the raw mean scores for each of the perceived neighborhood stressor domains. Given the different number of response scales, we standardized the individual items to $\mathrm{z}$ scores (mean $=0, \mathrm{SD}=1$ ) and summed the items representative of each perceived neighborhood construct and these scores are also presented in Table 1.

Because we were interested in a coordinated analysis, we chose constructs that were measured in at least two of the studies. Therefore, the following perceived neighborhood constructs were analyzed in relation to our cognitive outcomes: lack of safety (all studies), aesthetic quality (all studies), crime/violence (CATSLife, ESCAPE), and cohesion (ESCAPE, MIDUS). We used SAS version 9.4 (SAS Institute, 2013) for all descriptive statistics and to fit linear regressions in ESCAPE (PROC Reg) and multilevel models using maximum likelihood estimation in CATSLife and MIDUS (PROC Mixed). CATSLife and MIDUS include data from twins and siblings requiring a multilevel approach to account for family clustering and adjust for the reduction of standard errors due to data dependency. A random estimate representative of a family variable was therefore included to account for such clustering — only average fixed effects were of interest in this study and thus included in our presentation of results. In all models, we regressed the cognitive outcomes on the perceived neighborhood construct while first accounting for race and ethnicity, sex ( $0=$ female; $1=$ male), education (from $0=$ less than high school to $4=$ more than college), and age (centered at age 45 for all three studies). A subsequent model additionally adjusted for socioeconomic variables including marital status (from $0=$ married to $4=$ never married/ 
other), employment status ( $0=$ not employed; $1=$ employed $)$, and financial strain. Our final model additionally accounted for depressive symptoms and physical health (from $0=$ poor to $4=$ excellent). For the depressive symptoms and financial strain measures, we subtracted 1 from each response scale resulting in scale ranges with interpretable zero points (see Table 1). The final, fully adjusted, models are presented in this text, but the series of model adjustments are presented as Supplementary Tables 5 - 14. Because CATSLife combines two studies and adoption status in one of its substudies was shown to be associated with cognition (Ricker et al., 2018), we included adoption status ( $0=$ nonadopted, $1=$ adopted) as well as study sample as additional covariates in the CATSLife analyses (though those effects are not presented on the results for ease of comparability).

\section{Results}

Table 1 presents sample descriptions for each of the studies showing the diversity in demographic characteristics across the studies. Tables 2 through 5 show the estimated associations for the cognitive tasks in each study on each neighborhood domain. The estimates tended to not be statistically significant for the ESCAPE study, but the associations were comparable in direction and magnitude to CATSLife and MIDUS. Table 2 shows that lower perceived neighborhood safety predicted lower spatial performance in CATSLife ( $B=$ $-0.02(0.01), p<.05)$ and lower executive function in MIDUS $(B=-0.03(0.01), p<.0001)$. Lower perceptions of aesthetic quality predicted lower spatial performance $(B=-0.04$ $(0.01), p<.05)$ in CATSLife only as shown in Table 3. In MIDUS, lower perceived aesthetic quality was negatively associated with executive function in models accounting only for age, sex, race/ethnicity, and education $(B=-0.01(0.01), p<.01)$, but this association was no longer significant after accounting for self-reported health (see Supplementary Table 13). Table 4 shows that lower perceived neighborhood cohesion was suggestively associated with lower working memory performance in $\operatorname{ESCAPE}(B=-0.04(0.02), p<.10)$, and not related to executive function in MIDUS. Supplementary Table 14 shows that lower perceived cohesion was significantly associated with poorer executive function in MIDUS when accounting for age, sex, race/ethnicity and education only $(B=-0.02(0.01), p<.05)$, but this effect was no longer significant after accounting for marital status, financial strain and self-reported health. Lastly, Table 5 shows that more perceptions of crime were associated with lower performance in spatial abilities $(B=-0.02(<0.00), p<.01)$ in CATSLife, and similarly with lower performance in working memory in $\operatorname{ESCAPE~}(B=-0.04(0.02), n s)$, though that effect was not statistically significant.

Although age groups overlapped in all three studies (see Supplementary Figure 1 for a visual representation), there was variation in the ranges for each motivating a follow-up examination of age interactions to evaluate if the effect of neighborhoods on cognition were stronger in certain age groups. An interaction term of neighborhood domain by continuous age showed that the association between neighborhood domains and our cognitive outcomes did not vary by age (see Supplementary Tables $15-19$ ).

Lastly, as a way to contrast the results of these fluid, attention-demanding cognitive measures, we tested associations between the perceived neighborhood domains and measures of episodic memory (measured in all three studies) and verbal abilities (measured 
in CATSLife and ESCAPE), as non-fluid cognitive measures. As shown in Supplementary Tables $20-23$, we found no significant associations between the perceived neighborhood domains and vocabulary measures. The associations between perceived neighborhood domains and episodic memory were also not statistically significant with the exception of episodic memory on perceived neighborhood cohesion in MIDUS ( $B=-0.02(0.01), p$ $<.05)$.

\section{Discussion}

The role of self-reported neighborhood stressors on cognitive function in adulthood is not well understood and few studies have evaluated this association. Via a coordinated analysis of three studies of adults with comparable assessments of perceived neighborhoods, we found that perceived neighborhood stressors were associated with poorer performance on attention-demanding cognitive tasks. This study revealed an overall pattern whereby lower perceived neighborhood safety, lower perceived aesthetic quality, lower perceived neighborhood cohesion, and greater perceived neighborhood crime were more consistently associated with poorer performance on tasks of spatial and working memory, and executive functioning, but not perceptual speed tasks.

Through our coordinated analytic approach, we evaluated associations between comparable neighborhood domains in CATSLife, ESCAPE, and MIDUS and cognitive functioning outcomes related to fluid abilities, while accounting for almost identical demographic, socioeconomic, and health covariates. This approach allowed for a comparison of effects across studies. Because our outcome cognitive measures were $\mathrm{z}$ score standardized, the effects presented on our tables of results are indicative of effect sizes. For example, compared with other predictors in our models, scoring one unit higher in the perceived lack of neighborhood safety composite was comparable to the effect of being one year older in terms of performance on spatial abilities, working memory, and executive function. Our supplementary analyses showed that there was no interactive effect between age and perceived neighborhood stressors indicating independent effects on cognitive outcomes. We also found that the magnitude of association between specific neighborhood domains with cognition varied such that perceived lack of safety had the most consistent effect, followed by aesthetic quality, crime, and cohesion. The items used to capture lack of safety across the three studies pertained to participants' views that their neighborhood was not a safe place to be, such as not feeling safe to walk in their neighborhood during the day or views that personal belongings being stolen or torn up were a problem. One explanation for this being a robust effect may be because this domain has more direct implications for an individual's survival compared to aesthetics or sense of cohesion with neighbors, which have less immediate implications for a person's security, for example. From a stress process perspective, lack of neighborhood safety may activate greater perceptions of threat and thus a heighted emotional and physiological stress response (Cohen et al., 1995; Scott et al., 2018). Concurrently, these perceptions of threat may disrupt attentional processes such that attentional focus is directed toward the person's contextual threats as they may be continuously aware of their surroundings, resulting in lower attentional resources dedicated to the cognitive tasks at hand (Eysenck \& Calvo, 1992; Stawski et al., 2006). If such perceptions of threat are chronically activated, however, this can contribute to chronic stress 
and lead to neuropathological health outcomes (Smyth et al., 2013; Watkins, 2008).

Additional longitudinal studies investigating the long-term effects of perceived neighborhood safety on cognition, and the intermediate pathways, are needed to disentangle these effects.

Previous work on perceived neighborhood stressors and cognitive function focused on global measures of cognition or measures of crystallized abilities (Lee \& Waite, 2018; Zaheed et al., 2019). We extended this prior work by evaluating associations earlier in the lifespan starting at the cusp of midlife to older adulthood and testing associations with attentiondemanding, fluid cognitive abilities. Specifically, we focused on tasks of perceptual speed, spatial abilities, working memory, and executive function. We found that the associations with perceived neighborhood stressors were not significant for tasks of perceptual speed compared to spatial and working memory abilities. Tasks of speeded abilities are less demanding of attentional capacities and may be less sensitive to the cognitive demands of perceived neighborhood stressors than tasks of working memory, for example, that require greater processing resources. As a way of comparison, we also conducted supplementary analyses with episodic memory and vocabulary as two measures of crystallized abilities and found that perceived neighborhood stressors did not predict performance in these tasks with one exemption. In MIDUS, lower perceived neighborhood cohesion was associated with poorer episodic memory function $(B=-0.02(0.01), p<.05)$. This is related to findings by Zaheed and colleagues (2019) who reported a negative association between perceived neighborhood disorder and episodic memory in adults aged 51 and older from the Health and Retirement Study, which suggests that the effects of perceived neighborhoods on episodic memory may be discernable starting in late midlife. Furthermore, the associations between executive function and perceived neighborhood aesthetics and cohesion were significant in MIDUS before accounting for socioeconomic and health covariates indicating that these two constructs may mediate the observed associations later in midlife.

A limitation to acknowledge includes the cross-sectional nature of our analyses as the directionality of effects cannot be ascertained from these results. Longitudinal investigations with multiple assessments of perceived neighborhood stressors and cognitive function are necessary to disentangle directionality of effects. It is also important to consider objective neighborhood characteristics in relation to neighborhood perceptions and cognitive function. Lee and Waite (2018) previously reported that perceptions of neighborhoods were more important predictors of cognitive function than objective neighborhoods, but it has yet to be established whether objective neighborhoods may shape perceptions of neighborhood stressors which may then lead to reduced cognitive performance. A formal evaluation of this question was not possible in the current study given the lack of longitudinal perceived neighborhood data and objective neighborhoods in two of the studies included.

Participant address data were available for CATSLife and although participants were enrolled into their parent studies while living in the state of Colorado, geocoded participant address data showed that they now reside across the United States (Wadsworth et al., 2019). In an effort to contextualize these participants' neighborhoods, we leveraged national rankings of the Area Deprivation Index (ADI; University of Wisconsin School of Medicine Public Health, 2015). The ADI is a widely used marker of socioeconomic deprivation within 
a neighborhood that is based on multiple Census indices where higher values indicate higher deprivation (Singh, 2003). These data showed that CATSLife participants lived across a wide range of contexts with the median ADI in the $30^{\text {th }}$ national percentile (observed range: 1 - 100 with higher percentiles indicating more deprivation). Data collected in the ESCAPE study originated in an urban setting in Bronx, New York. Incorporating ADI data from the single zip code within which participants resided, we observed that this neighborhood fell in the top $8^{\text {th }}$ to $9^{\text {th }}$ decile of relative deprivation within the state of New York and the top 80 percent nationally, indicating greater levels of socioeconomic deprivation within this sample. Though geocoded data for MIDUS were not available, this was designed to be a nationally representative sample of the United States that likely covers a wide range of neighborhood contexts. Future studies evaluating the role of these objective contexts on perceived neighborhood stressors will additionally contribute to a better understanding of the associations found in this study.

The central goal of this study was to coordinate across three studies with multi-domain assessments of perceived neighborhood stressors to evaluate the consistency of associations with attention-demanding fluid cognitive abilities starting at the cusp of midlife. A strength of this approach is that we were able to compare effect sizes across three seemingly heterogeneous studies of adults and found that the direction and the size of the estimated effects were similar across all three studies. The associations tended to be statistically reliable for the CATSLife and MIDUS studies, but not for the ESCAPE study, which was smallest among the three samples. Also, the cognitive assessments in MIDUS were obtained over the phone and CATSLife is part of a longitudinal study involving the repeated return of participants into the laboratory for assessments. In comparison, ESCAPE participants were new to the study protocol and were likely unfamiliar with cognitive assessments in the laboratory, highlighting the possibility that differences in the familiarity of testing contexts might have also influenced our results. Because the central interest in studies evaluating neighborhood-related factors and cognition are the influences of the contexts in which individuals are embedded, attention should be directed toward the measurement settings used in future studies.

In conclusion, this study is the first to evaluate the association between specific perceived neighborhood stressors and cognitive performance in specific tasks of fluid and executive abilities in three studies starting at the cusp of midlife. We coordinated an analysis across CATSLife, ESCAPE, and MIDUS and found that perceived lack of neighborhood safety, lower perceived aesthetic quality, lower perceptions of cohesion, and greater perceptions of crime are systematically associated with lower performance in tasks of spatial abilities, working memory, and executive function. These results highlight the significance of the psychosocial neighborhood context for cognitive health in adulthood.

\section{Supplementary Material}

Refer to Web version on PubMed Central for supplementary material. 


\section{Acknowledgements}

Funding: The CATSLife is supported by a grant and administrative supplement from the NIA (AG046938 and AG046938-03S1; Reynolds \& Wadsworth, MPIs). The CAP and the LTS have been funded across the years by numerous sources, including a CRCW grant from the University of Colorado; National Institutes of Health grants HD010333, HD18426, MH43899, HD036773, DA05131, DA011015, DA046064 and DA042755; the Spencer and William T. Grant Foundations; and the John D. and Catherine T. MacArthur Foundation. The ECF-LTS is currently supported by MH063207.

The ESCAPE study was supported by National Institute of Health (NIH) grants R01 AG039409, R01 AG042595, P01 AG03949, CTSA 1UL1TR001073 from the National Center for Advancing Translational Sciences (NCATS), the Leonard and Sylvia Marx Foundation, and the Czap Foundation.

Publicly available data from the MIDUS study was used for this research. Since 1995 the MIDUS study has been funded by the following: John D. and Catherine T. MacArthur Foundation Research Network and the National Institute on Aging (P01-AG020166 \& U19-AG051426).

During data analysis and the preparation of the manuscript, Elizabeth Muñoz was partly supported by NIA F32AG056134 and by grant, P2CHD042849, Population Research Center, awarded to the Population Research Center at The University of Texas at Austin by the Eunice Kennedy Shriver National Institute of Child Health and Human Development.

The content is solely the responsibility of the authors and does not necessarily represent the official views of the National Institutes of Health.

\section{References}

Agrigoroaei S, \& Lachman ME (2011). Cognitive functioning in midlife and old age: Combined effects of psychosocial and behavioral factors. The Journals of Gerontology Series B: Psychological Sciences and Social Sciences, 66B, i130-i140. 10.1093/geronb/gbr017

Aneshensel CS, Ko MJ, Chodosh J, \& Wight RG (2011). The urban neighborhood and cognitive functioning in late middle age. Journal of Health and Social Behavior, 52(2), 163-179.

10.1177/0022146510393974 [PubMed: 21673145]

Aneshensel CS, Wight RG, Miller-Martinez D, Botticello AL, Karlamangla AS, \& Seeman TE (2007). Urban neighborhoods and depressive symptoms among older adults. The Journals of Gerontology Series B: Psychological Sciences and Social Sciences, 62(1), S52-S59.

Augustin T, Glass TA, James BD, \& Schwartz BS (2008). Neighborhood psychosocial hazards and cardiovascular disease: The Baltimore memory study. American Journal of Public Health, 98(9), 1664-1670. 10.2105/AJPH.2007.125138 [PubMed: 18633086]

Bäckman L, \& Small BJ (2007). Cognitive deficits in preclinical Alzheimer's disease and vascular dementia: Patterns of findings from the Kungsholmen Project. Physiology \& Behavior, 92(1-2), 8086. 10.1016/j.physbeh.2007.05.014 [PubMed: 17573076]

Boardman JD, Barnes LL, Wilson RS, Evans DA, \& de Leon CFM (2012). Social disorder, APOE-E4 genotype, and change in cognitive function among older adults living in Chicago. Social Science \& Medicine, 74(10), 1584-1590. 10.1016/j.socscimed.2012.02.012 [PubMed: 22465377]

Bosma H (2001). Neighborhood socioeconomic status and all-cause mortality. American Journal of Epidemiology, 153(4), 363-371. 10.1093/aje/153.4.363 [PubMed: 11207154]

Choi YJ, \& Matz-Costa C (2018). Perceived neighborhood safety, social cohesion, and psychological health of older adults. The Gerontologist, 58(1), 196-206. 10.1093/geront/gnw187 [PubMed: 28082279]

Clarke PJ, Weuve J, Barnes L, Evans DA, \& Mendes de Leon CF (2015). Cognitive decline and the neighborhood environment. Annals of Epidemiology, 25(11), 849-854. 10.1016/ j.annepidem.2015.07.001 [PubMed: 26253697]

Cohen S, Kamarck T, \& Mermelstein R (1983). A global measure of perceived stress. Journal of Health and Social Behavior, 24(4), 385-396. 10.2307/2136404 [PubMed: 6668417]

Cohen S, Kessler RC, \& Gordon LU (1995). Measuring stress: A guide for health and social scientists: Vol. xii. Oxford University Press. 
Cohen S, \& Wills TA (1985). Stress, social support, and the buffering hypothesis. Psychological Bulletin, 98(2), 310-357. 10.1037/0033-2909.98.2.310 [PubMed: 3901065]

Conway ARA, Cowan N, Bunting MF, Therriault DJ, \& Minkoff SRB (2002). A latent variable analysis of working memory capacity, short-term memory capacity, processing speed, and general fluid intelligence. Intelligence, 30(2), 163-183. 10.1016/S0160-2896(01)00096-4

DeFries JC, Ashton GC, Johnson RC, Kuse AR, McClearn GE, Mi MP, Rashad MN, Vandenberg SG, \& Wilson JR (1978). The Hawaii family study of cognition: A reply. Behavior Genetics, 8(3), 281-288. 10.1007/BF01072831 [PubMed: 687321]

DeFries JC, Plomin R, Vandenberg SG, \& Kuse AR (1981). Parent-offspring resemblance for cognitive abilities in the Colorado Adoption Project: Biological, adoptive, and control parents and one-yearold children. Intelligence, 5(3), 245-277. 10.1016/S0160-2896(81)80012-8

Diez Roux AV, \& Mair C (2010). Neighborhoods and health. Annals of the New York Academy of Sciences, 1186(1), 125-145. 10.1111/j.1749-6632.2009.05333.x [PubMed: 20201871]

Engle RW, Tuholski SW, Laughlin JE, \& Conway ARA (1999). Working memory, short-term memory, and general fluid intelligence: A latent-variable approach. Journal of Experimental Psychology: General, 128(3), 309-331. 10.1037/0096-3445.128.3.309 [PubMed: 10513398]

Estrella ML, Durazo-Arvizu RA, Gallo LC, Isasi CR, Perreira KM, Vu T-HT, Vasquez E, Sachdeva S, Zeng D, Llabre MM, Tarraf W, González HM, Daviglus ML, \& Lamar M (2020). Associations between perceived neighborhood environment and cognitive function among middle-aged and older women and men: Hispanic Community Health Study/Study of Latinos Sociocultural Ancillary Study. Social Psychiatry and Psychiatric Epidemiology. 10.1007/s00127-019-01829-0

Eysenck MW, \& Calvo MG (1992). Anxiety and performance: The processing efficiency theory. Cognition \& Emotion, 6(6), 409-434. 10.1080/02699939208409696

Fisher KJ, Li F, Michael Y, \& Cleveland M (2004). Neighborhood-level influences on physical activity among older adults: A multilevel analysis. Journal of Aging and Physical Activity, 12(1), 45-63. [PubMed: 15211020]

Glass TA, Bandeen-Roche K, McAtee M, Bolla K, Todd AC, \& Schwartz BS (2009). Neighborhood psychosocial hazards and the association of cumulative lead dose with cognitive function in older adults. American Journal of Epidemiology, 169(6), 683-692. 10.1093/aje/kwn390 [PubMed: 19155330]

Hofer SM, \& Piccinin AM (2009). Integrative data analysis through coordination of measurement and analysis protocol across independent longitudinal studies. Psychological Methods, 14(2), 150-164. 10.1037/a0015566 [PubMed: 19485626]

Hughes ML, Agrigoroaei S, Jeon M, Bruzzese M, \& Lachman ME (2018). Change in cognitive performance from midlife into old age: Findings from the Midlife in the United States (MIDUS) study. Journal of the International Neuropsychological Society, 1-16. 10.1017/ S1355617718000425

Krueger KR, Wilson RS, Kamenetsky JM, Barnes LL, Bienias JL, \& Bennett DA (2009). Social engagement and cognitive function in old age. Experimental Aging Research, 35(1), 45-60. 10.1080/03610730802545028 [PubMed: 19173101]

Lachman ME, \& Tun PA (2008). Cognitive testing in large-scale surveys: Assessment by telephone In Handbook of cognitive aging: Interdisciplinary perspectives (pp. 506-523). Sage Publications, Inc. 10.4135/9781412976589.n30

Lang IA, Llewellyn DJ, Langa KM, Wallace RB, Huppert FA, \& Melzer D (2008). Neighborhood deprivation, individual socioeconomic status, and cognitive function in older people: Analyses from the English Longitudinal Study of Ageing. Journal of the American Geriatrics Society, 56(2), 191-198. 10.1111/j.1532-5415.2007.01557.x [PubMed: 18179489]

Lazarus RS, \& Folkman S (1984). Stress, appraisal, and coping. Springer Pub. Co.

Lee H, \& Waite LJ (2017). Cognition in context: The role of objective and subjective measures of neighborhood and household in cognitive functioning in later life. The Gerontologist. 10.1093/ geront/gnx050

Lupien SJ, \& Lepage M (2001). Stress, memory, and the hippocampus: Can't live with it, can't live without it. Behavioural Brain Research, 127(1-2), 137-158. 10.1016/S0166-4328(01)00361-8 [PubMed: 11718889] 
Malambo P, Kengne AP, Lambert EV, De Villiers A, \& Puoane T (2017). Does physical activity mediate the association between perceived neighborhood aesthetics and overweight/obesity among South African adults living in selected urban and rural communities? Journal of Physical Activity \& Health, 14(12), 925-932. 10.1123/jpah.2016-0147 [PubMed: 28682650]

McCoy DC, Raver CC, \& Sharkey P (2015). Children's cognitive performance and selective attention following recent community violence. Journal of Health and Social Behavior, 56(1), 19-36. 10.1177/0022146514567576 [PubMed: 25663176]

McEwen BS, \& Sapolsky RM (1995). Stress and cognitive function. Current Opinion in Neurobiology, 5(2), 205-216. 10.1016/0959-4388(95)80028-X [PubMed: 7620309]

Mujahid MS, Diez Roux AV, Morenoff JD, \& Raghunathan T (2007). Assessing the measurement properties of neighborhood scales: From psychometrics to ecometrics. American Journal of Epidemiology, 165(8), 858-867. 10.1093/aje/kwm040 [PubMed: 17329713]

Muthén LK, \& Muthén BO (1998). Mplus User's Guide. (Seventh Edition). Muthén \& Muthén.

Parkes A, Kearns A, \& Atkinson R (2002). What makes people dissatisfied with their neighbourhoods? Urban Studies, 39(13), 2413-2438. 10.1080/0042098022000027031

PROMIS Health Organization. (2012a). Emotional distress- anger- short form 8a. Unpublished instrument Retrieved from https://www.assessmentcenter.net

PROMIS Health Organization. (2012b). Global health scale- short form. Unpublished instrument Retrieved from https://www.assessmentcenter.net

Pruchno RA, Wilson-Genderson M, \& Cartwright FP (2012). The texture of neighborhoods and disability among older adults. The Journals of Gerontology Series B: Psychological Sciences and Social Sciences, 67B(1), 89-98. 10.1093/geronb/gbr131

Ricker AA, Corley R, DeFries JC, Wadsworth SJ, \& Reynolds CA (2018). Examining the influence of perceived stress on developmental change in memory and perceptual speed for adopted and nonadopted individuals. Developmental Psychology, 54(1), 138-150. 10.1037/dev0000329 [PubMed: 28981301]

Ryff C, Almeida DM, Ayanian J, Carr DS, Cleary PD, Coe C, Davidson R, Krueger RF, Lachman ME, Marks NF, Mroczek DK, Seeman T, Seltzer MM, Singer BH, Sloan RP, Tun PA, Weinstein M, \& Williams D (2007). Midlife in the United States (MIDUS 2), 2004-2006. [Data set]. Ann Arbor, MI: ICPSR - Interuniversity Consortium for Political and Social Research 10.3886/ ICPSR04652.V7

Sallis JF, Bowles HR, Bauman A, Ainsworth BE, Bull FC, Craig CL, Sjöström M, De Bourdeaudhuij I, Lefevre J, Matsudo V, \& others (2009). Neighborhood environments and physical activity among adults in 11 countries. American Journal of Preventive Medicine, 36(6), 484-490. [PubMed: 19460656]

Sallis JF, \& DeBourdeaudhuij I (2004). International Physical Activity and the Environment Network (IPEN). https://www.ipenproject.org/

Salthouse TA (1996). The processing-speed theory of adult age differences in cognition. Psychological Review, 103(3), 403-428. [PubMed: 8759042]

Sampson RJ (2003). The neighborhood context of well-being. Perspectives in Biology and Medicine, 46(3), S53-S64. 10.1353/pbm.2003.0073 [PubMed: 14563074]

Sampson RJ, Morenoff JD, \& Gannon-Rowley T (2002). Assessing "neighborhood effects": Social processes and new directions in research. Annual Review of Sociology, 28(1), 443-478. 10.1146/ annurev.soc.28.110601.141114

SAS Institute. (2013). SAS (Version 9.4). SAS Institute, Inc.

Scott SB, Graham-Engeland JE, Engeland CG, Smyth JM, Almeida DM, Katz MJ, Lipton RB, Mogle JA, Munoz E, Ram N, \& Sliwinski MJ (2015). The Effects of Stress on Cognitive Aging, Physiology and Emotion (ESCAPE) project. BMC Psychiatry, 15(1), 146 10.1186/ s12888-015-0497-7 [PubMed: 26138700]

Scott SB, Munoz E, Mogle JA, Gamaldo AA, Smyth JM, Almeida DM, \& Sliwinski MJ (2018). Perceived neighborhood characteristics predict severity and emotional response to daily stressors. Social Science \& Medicine, 200, 262-270. 10.1016/j.socscimed.2017.11.010 [PubMed: 29191514] 
Sliwinski MJ, Mogle JA, Hyun J, Munoz E, Smyth JM, \& Lipton RB (2016). Reliability and validity of ambulatory cognitive assessments. Assessment. 10.1177/1073191116643164

Smyth J, Zawadzki M, \& Gerin W (2013). Stress and disease: A structural and functional analysis: chronic stress and health. Social and Personality Psychology Compass, 7(4), 217-227. 10.1111/ $\operatorname{spc} 3.12020$

Stawski RS, Sliwinski MJ, \& Smyth JM (2006). Stress-related cognitive interference predicts cognitive function in old age. Psychology and Aging, 21(3), 535-544. 10.1037/0882-7974.21.3.535 [PubMed: 16953715]

Su M, Tan Y, Liu Q, Ren Y, Kawachi I, Li L, \& Lv J (2014). Association between perceived urban built environment attributes and leisure-time physical activity among adults in Hangzhou, China. Preventive Medicine, 66, 60-64. 10.1016/j.ypmed.2014.06.001 [PubMed: 24929197]

Tun PA, \& Lachman ME (2006). Telephone assessment of cognitive function in adulthood: The Brief Test of Adult Cognition by Telephone. Age and Ageing, 35(6), 629-632. 10.1093/ageing/afl095 [PubMed: 16943264]

Wadsworth SJ, Corley RP, Munoz E, Trubenstein BP, Knaap E, DeFries JC, Plomin R, Reynolds CA, \& Team T. Catsl. (2019). CATSLife: A study of lifespan behavioral development and cognitive functioning. Twin Research and Human Genetics, 1-12. 10.1017/thg.2019.49 [PubMed: 30661510]

Waters GS, \& Caplan D (2003). The reliability and stability of verbal working memory measures. Behavior Research Methods, Instruments, \& Computers, 35(4), 550-564. 10.3758/BF03195534

Watkins ER (2008). Constructive and unconstructive repetitive thought. Psychological Bulletin, 134, 163-206. 10.1037/0033-2909.134.2.163 [PubMed: 18298268]

Wechsler D (1996). Wechsler Adult Intelligence Scale-III (WAIS-III). Psychological Corp.

Wight RG, Aneshensel CS, Miller-Martinez D, Botticello AL, Cummings JR, Karlamangla AS, \& Seeman TE (2006). Urban neighborhood context, educational attainment, and cognitive function among older adults. American Journal of Epidemiology, 163(12), 1071-1078. 10.1093/aje/kwj176 [PubMed: 16707655]

Wu Y-T, Prina AM, \& Brayne C (2015). The association between community environment and cognitive function: A systematic review. Social Psychiatry and Psychiatric Epidemiology, 50(3), 351-362. 10.1007/s00127-014-0945-6 [PubMed: 25087013]

Zaheed AB, Sharifian N, Kraal AZ, Sol K, Hence A, \& Zahodne LB (2019). Unique effects of perceived neighborhood physical disorder and social cohesion on episodic memory and semantic fluency. Archives of Clinical Neuropsychology, 34(8), 1346-1355. 10.1093/arclin/acy098 [PubMed: 30715092]

Zeki Al Hazzouri A, Haan MN, Osypuk T, Abdou C, Hinton L, \& Aiello AE (2011). Neighborhood socioeconomic context and cognitive decline among older Mexican Americans: Results from the Sacramento Area Latino Study on Aging. American Journal of Epidemiology, 174(4), 423-431. 10.1093/aje/kwr095 [PubMed: 21715645] 
- Neighborhood stressors were associated with poorer cognitive performance.

- $\quad$ Perceived neighborhood stressors were associated with lower performance in spatial abilities, working memory, and executive function, but not perceptual speed.

- $\quad$ Effects on cognition were more consistent for lower perceived neighborhood safety followed by lower perceived aesthetic quality, greater perceived neighborhood crime, and lower perceived neighborhood cohesion. 


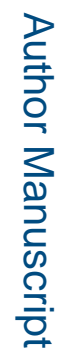

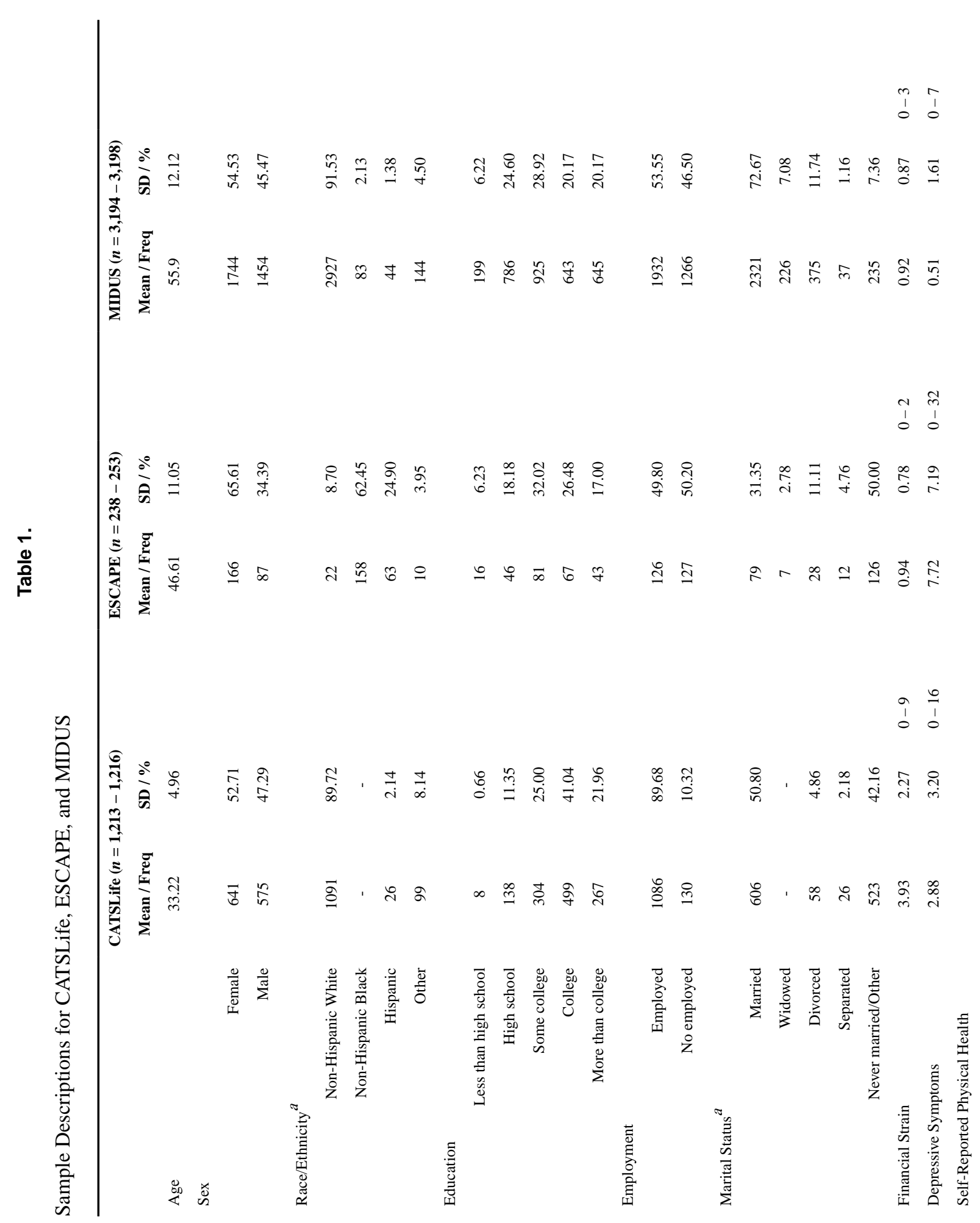




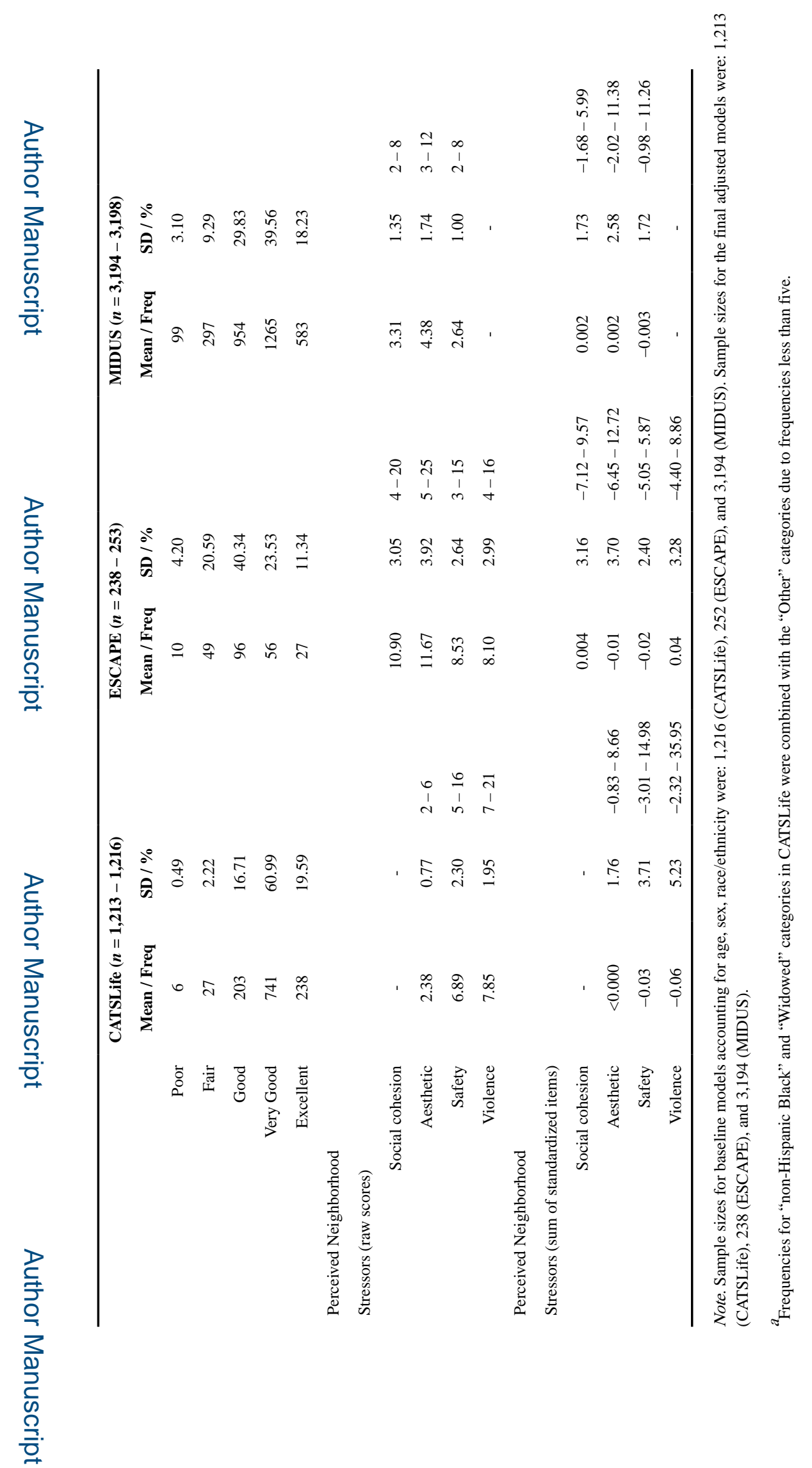

Health Place. Author manuscript; available in PMC 2021 November 01. 


\section{을

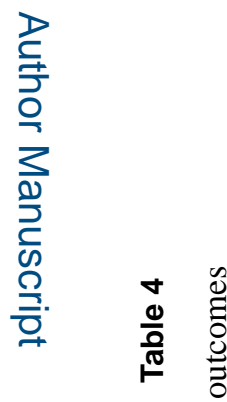

$$
\text { 竞 }
$$

(1)

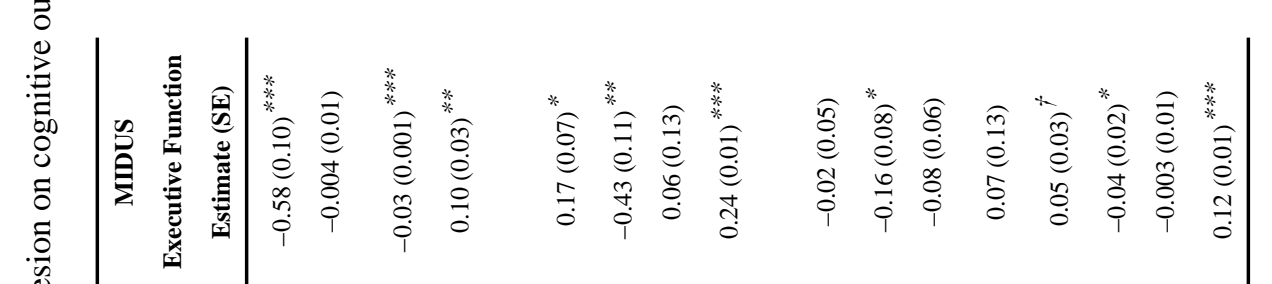

西 
西 